\title{
Immunohistochemical staining of ERG and SOX9 as potential biomarkers of docetaxel response in patients with metastatic castration-resistant prostate cancer
}

\author{
Wan Song ${ }^{1}$, Ghee Young Kwon ${ }^{2}$, Jeong Hoon $\mathrm{Kim}^{3}$, Joung Eun Lim ${ }^{4}$, Hwang Gyun \\ Jeon ${ }^{1}$, Seong Il Seo ${ }^{1}$, Seong Soo Jeon ${ }^{1}$, Han Yong Choi ${ }^{1}$, Byong Chang Jeong ${ }^{1}$, \\ Hyun Moo Lee ${ }^{1}$ \\ ${ }^{1}$ Department of Urology, Samsung Medical Center, Sungkyunkwan University School of Medicine, Seoul, Korea \\ ${ }^{2}$ Department of Pathology, Samsung Medical Center, Sungkyunkwan University School of Medicine, Seoul, Korea \\ ${ }^{3}$ Department of Molecular Biology, Samsung Advanced Institute for Health Science and Technology, Sungkyunkwan University \\ School of Medicine, Seoul, Korea \\ ${ }^{4}$ Department of Urology, Samsung Biomedical Research Institute, Samsung Medical Center, Seoul, Korea \\ Correspondence to: Byong Chang Jeong, email: bc2.jung@samsung.com \\ Hyun Moo Lee, email: hyunmoo.lee@samsung.com \\ Keywords: biomarker, docetaxel, ERG, prostate cancer, SOX9
}

Received: July 26, $2016 \quad$ Accepted: October 12, $2016 \quad$ Published: November 16, 2016

\section{ABSTRACT}

We aimed to evaluate ERG and SOX9 as potential biomarkers of docetaxel response in metastatic castration-resistant prostate cancer (mCRPC) patients. Seventy-one mCRPC patients were evaluated. Tissue microarrays were constructed and immunohistochemistry was performed. Treatment response was assessed by prostate specific antigen (PSA) response rate, PSA progression-free survival (PSAPFS), clinical/radiologic PFS (C/R-PFS) and overall survival (OS). ERG and SOX9 were found in $13(18.3 \%)$ and $62(87.3 \%)$ patients, respectively. ERG-positive had lower PSA response rates than negative $(15.4 \%$ vs $62.1 \%, p=0.004)$, and SOX9 showed a same trend $(46.8 \%$ vs $100.0 \%, p=0.003)$. ERG positivity correlated with a lower PSA-PFS (3.2 mos vs 7.4 mos, $p<0.001$ ), C/R-PFS (3.8 mos vs 9.0 mos, $p$ $<0.001$ ) and OS (10.8 mos vs 21.4 mos, $p<0.001)$. SOX9 positivity also showed a lower PSA-PFS, C/R-PFS and OS $(p=0.006, p=0.012$ and $p=0.023$, respectively). On multivariate analysis, ERG positivity was a significant risk factor for a lower PSA-PFS, C/R-PFS and OS ( $p<0.001, p<0.001$ and $p=0.001$, respectively). SOX9 expression was also a risk factor for a lower PSA-PFS, C/R-PFS and OS $(p=0.018, p=0.025$ and $p=0.047$, respectively). These findings indicate that ERG and SOX9 is potential biomarkers for prediction to docetaxel treatment in mCRPC patients.

\section{INTRODUCTION}

Treatment of metastatic castration-resistant prostate cancer (mCRPC) is a major clinical challenge. Patients with $\mathrm{mCRPC}$ have a poor prognosis with an expected survival time less than 2 years [1]. Docetaxel-based chemotherapy is recommended as first-line standard of care for mCRPC [2] based on the results of two phase III studies (Southwest Oncology Group [SWOG] 9916 and Taxotere $[\mathrm{Tax}] 327)$ that demonstrated a significant survival benefit $[3,4]$. However, most of the patients eventually developed treatment resistance and experienced treatment-related toxicity $[5,6]$, thereby underscoring the need for a biomarker for prediction to docetaxel treatment.

Recently, several molecular studies have elucidated the mechanisms for docetaxel resistance and have broadened our understanding of mCRPC [7-9]. E26 transformation-specific (ETS)-related gene (ERG) expression was increased 30 to 80 times above normal levels in approximately $50 \%$ of prostate cancer $[10,11]$. An in vitro study showed that overexpressed ERG binds to microtubules and alters their dynamics. This also inhibits drug-target engagement, thus leading to docetaxel resistance $[10,12]$. In addition, two studies examined the 
function of ERG and identified SRY-related HMG box (SOX) 9 as an important downstream effector of ERG [13, 14]. Therefore, expression of ERG and SOX9 in mCRPC patients might influence on the treatment outcomes.

In this study, we constructed tissue microarrays (TMAs) using prostate biopsy samples and carried out immunohistochemistry (IHC) analyses to evaluate the clinical utility of ERG and SOX9 as potential biomarkers of docetaxel response in $\mathrm{mCRPC}$ patients.

\section{RESULTS}

The baseline characteristics of 71 patientswith mCRPC who underwent docetaxel treatment are presented in Table 1. At the time of diagnosis, the mean age and prostate specific antigen (PSA) were 64.9 (7.5, 49.0-88.0) years and $775.7(1597.0,4.6-7539.3) \mathrm{ng} / \mathrm{ml}$, respectively. The mean duration of androgen deprivation therapy (ADT) use prior to docetaxel treatment was 28.6 (20.6, 3.3-94.3) months, and the mean PSA nadir after ADT was 5.1 (11.8, 0.01-65.66) ng/ml. Forty-seven (66.2\%) patients had a high metastatic burden at the time of docetaxel treatment. During a mean follow-up period of 21.6 (14.7, 3.2-86.8) months post-docetaxel treatment, all patients developed both PSA and C/R progression, 54 (76.1\%) of whom died. When patients were divided depending on ERG expression, baseline characteristics of MCRPC patients were not significantly different except initial PSA.

Of the total 71 patients, ERG was positive in 13 $(18.3 \%)$ patients and $62(87.3 \%)$ patients were SOX9positive. All patients with positive ERG expression as detected via IHC also showed SOX9 positivity. However, in patients negative for ERG expression, 49 (84.5\%) were SOX9-positive and $9(15.5 \%)$ patients were negative. The correlation of IHC results with ERG and SOX9 expression is depicted in Figure 1.

Figure 2 shows the PSA response rate according to ERG and SOX9 IHC results. ERG-positive patients had a lower PSA response rate than negative patients $(15.4 \%$ vs $62.1 \%, p=0.004)$. SOX9 also presented a same trend (46.8\% vs $100.0 \%, p=0.003)$. The PSA-PFS, C/R-PFS and $\mathrm{OS}$ values estimated using the Kaplan-Meier method and the results of the log-rank test are presented in Figure 3. There were significant differences in the PSA-PFS, $\mathrm{C} / \mathrm{R}-\mathrm{PFS}$ and OS according to ERG expression (Figure 3A) (all $p<0.001$, respectively). The median PSA-PFS was 3.2 months in ERG-positive patients and 7.4 months in negative patients. The median C/R-PFS and OS were 3.8 months and 10.8 months in ERG-positive patients, and 9.0 months and 21.4 months in ERG-negative patients, respectively. In addition, a positive SOX9 result was also correlated with a lower PSA-PFS, C/R-PFS and OS than a negative SOX9 result (Figure 3B). The median PSA-PFS and C/R-PFS were 7.1 months and 7.4 months in SOX9positive patients, and 9.3 months and 11.0 months in SOX9-negative patients, respectively. The median OS was
19.7 months in SOX9-positive patients but not reached to median in SOX9 negative patients. When we analyzed the patients in 3 subgroups according to the combined effects of ERG and SOX9, the presence of both ERG and SOX9 positivity was significantly associated with a lower PSA-PFS, C/R-PFS and OS (Figure 3C) (all $p<0.001$, respectively).

Table 2 shows the Cox proportional hazard regression analysis for the PSA-PFS, C/R-PFS and OS in all $71 \mathrm{mCRPC}$ patients. On multivariate analysis, ERG positivity was significantly associated with a lower PSAPFS ( $p<0.001$, hazard ratio (HR): $6.00,95 \%$ confidence interval (CI): 2.96-12.16), C/R-PFS ( $p<0.001$, HR: 5.50, 95\% CI: 2.68-11.29) and OS ( $p=0.001$, HR: 3.31, 95\% CI: 1.66-6.64). In addition, SOX9 was a significant risk factor for a decreased PSA-PFS ( $p=0.018$, HR: 2.75, 95\% CI: 1.19-6.32), C/R-PFS ( $p=0.025$, HR: $2.44,95 \%$ CI: $1.12-5.30)$ and OS ( $p=0.047$, HR: $4.30,95 \% \mathrm{CI}$ : 1.02-18.16). High metastatic volume was significantly associated with a lower C/R-PFS ( $p=0.003$, HR: 2.31 , 95\% CI: $1.32-4.04)$ and OS ( $p=0.003$, HR: $2.88,95 \%$ CI: 1.44-5.76), but not the PSA-PFS.

\section{DISCUSSION}

In this study, $18.3 \%$ and $87.3 \%$ of patients with mCRPC showed positive ERG and SOX9 expression per IHC analysis, respectively. When examining their associations with clinical outcomes, ERG and SOX9 were significant risk factors for lower PSA-PFS, C/R-PFA and OS after docetaxel treatment. In addition, their effects on docetaxel response were even more exaggerated when analyzed in the 3 subgroups. These results suggest that ERG and SOX9 is potential biomarkers for prediction to docetaxel treatment in mCRPC patients. To the best of our knowledge, our study is the first study to analyze the correlation between ERG and SOX9 as measured by IHC and docetaxel response in $\mathrm{MCRPC}$ patients.

Recent studies showed that ERG overexpression occurs in at least $50 \%$ of prostate cancer cases as a result of gene fusion, with a TMPRESS2-ERG rearrangement being the most common form $[10,15,16]$. In a study by Galletti et al [10], eleven (32.4\%) patients were positive for the TMPRESS2-ERG fusion, and PSA response to docetaxel was associated with circulation tumor cell (CTC) ERG expression in $34 \mathrm{mCRPC}$ patients (positive; $45 \%$ vs negative; $79 \%, \mathrm{p}=0.056)$. In addition, Reig et al [15] reported that $8(16 \%)$ of 50 docetaxel-treated mCRPC patients had CTCs positive for the TMPRESS2ERG fusion. This finding was also significantly associated with a lower PSA response, PSA-PFS, C/R-PFS and OS. Furthermore, in the 25 docetaxel-treated mCRPC patients who had tissue samples available, TMPRESS2-ERG was found to be positive in $14(56 \%)$ patients and associated with lower PSA-PFS, but not C/R-PFS. 
Table 1: Baseline characteristics of mCRPC patients

\begin{tabular}{|c|c|c|c|c|}
\hline \multirow{2}{*}{ Variables } & \multirow{2}{*}{ Total } & \multicolumn{2}{|c|}{ Immunohistochemistry } & \multirow{2}{*}{$\boldsymbol{P}$} \\
\hline & & ERG(+) & ERG(-) & \\
\hline No. of patients & $71(100)$ & $13(18.3)$ & $58(81.7)$ & \\
\hline Age at diagnosis, years & $\begin{array}{l}64.9 \pm 7.5[64.0 \\
\quad 49.0-88.0]\end{array}$ & $\begin{array}{l}63.5 \pm 10.6[62.0 \\
\quad 49.0-88.0]\end{array}$ & $\begin{array}{l}65.2 \pm 6.6[65.5 \\
\quad 52.0-78.0]\end{array}$ & 0.450 \\
\hline Gleason score at diagnosis & & & & 0.346 \\
\hline 7 & $9(12.7)$ & $1(7.7)$ & $8(13.8)$ & \\
\hline 8 & $19(26.8)$ & $3(23.1)$ & $16(27.6)$ & \\
\hline 9 & $32(45.1)$ & $6(46.1)$ & $26(44.8)$ & \\
\hline 10 & $11(15.5)$ & $3(23.1)$ & $8(13.8)$ & \\
\hline Initial PSA, ng/ml & $\begin{array}{c}775.7 \pm 1597.0 \\
{[166.6,4.6-7539.3]}\end{array}$ & $\begin{array}{c}170.9 \pm 174.8 \\
{[134.2,8.7-551.0]}\end{array}$ & $\begin{array}{c}916.1 \pm 1743.6 \\
{[225.9,4.6-7539.3]}\end{array}$ & 0.003 \\
\hline PSA nadir after ADT & $\begin{array}{l}5.1 \pm 11.8[1.0,0.01- \\
65.66]\end{array}$ & $\begin{array}{l}6.5 \pm 8.4[2.0,0.07- \\
19.52]\end{array}$ & $\begin{array}{l}4.7 \pm 12.5[0.9,0.01- \\
65.66]\end{array}$ & 0.624 \\
\hline Metastatic status before docetaxel treatment & & & & 0.798 \\
\hline Low volume & $24(33.8)$ & $4(30.8)$ & $20(34.5)$ & \\
\hline High volume & $47(66.2)$ & $9(69.2)$ & $38(65.5)$ & \\
\hline Type of local treatment & & & & 0.358 \\
\hline None & $62(87.3)$ & $12(92.3)$ & $50(86.2)$ & \\
\hline Prostatectomy & $3(4.2)$ & $1(7.7)$ & $2(3.5)$ & \\
\hline HIFU & $6(8.5)$ & 0 & $6(10.3)$ & \\
\hline ADT duration prior to docetaxel treatment & $\begin{array}{l}28.6 \pm 20.6[22.9 \\
\quad 3.3-94.3]\end{array}$ & $\begin{array}{l}22.5 \pm 18.3[17.2 \\
\quad 3.3-66.7]\end{array}$ & $\begin{array}{l}30.0 \pm 20.9[23.7 \\
\quad 6.3-94.3]\end{array}$ & 0.240 \\
\hline No. of docetaxel regimens & $\begin{array}{l}6.9 \pm 4.0[6.0,3.0- \\
17.0]\end{array}$ & $\begin{array}{l}7.3 \pm 3.3[8.0,3.0- \\
12.0]\end{array}$ & $\begin{array}{c}6.8 \pm 4.1[5.0,3.0- \\
17.0]\end{array}$ & 0.707 \\
\hline \multicolumn{5}{|l|}{ Follow-up, months } \\
\hline From initial diagnosis to docetaxel treatment & $\begin{array}{l}31.3 \pm 21.6[25.7 \\
2.1-94.0]\end{array}$ & $\begin{array}{l}20.5 \pm 15.0[18.3 \\
\quad 2.1-41.0]\end{array}$ & $\begin{array}{l}33.8 \pm 22.2[26.4 \\
3.4-94.0]\end{array}$ & 0.045 \\
\hline $\begin{array}{l}\text { From docetaxel treatment to death or last } \\
\text { visit }\end{array}$ & $\begin{array}{l}21.6 \pm 14.7[17.6 \\
3.2-86.8]\end{array}$ & $\begin{array}{c}12.5 \pm 7.8[10.8,3.2- \\
26.2]\end{array}$ & $\begin{array}{c}23.6 \pm 15.1[19.6 \\
4.0-86.8]\end{array}$ & 0.013 \\
\hline Overall & $\begin{array}{c}52.9 \pm 27.2[5.3- \\
126.4]\end{array}$ & $\begin{array}{c}33.0 \pm 19.8[31.1 \\
5.3-63.1]\end{array}$ & $\begin{array}{c}57.4 \pm 26.8[51.7 \\
21.1-126.4]\end{array}$ & 0.003 \\
\hline Type of post-chemotherapy treatment & & & & 0.423 \\
\hline None & $51(71.9)$ & $9(69.2)$ & $42(72.5)$ & \\
\hline Abiraterone only & $4(5.6)$ & $2(15.4)$ & $2(3.4)$ & \\
\hline Cabazitaxel only & $3(4.2)$ & $1(7.7)$ & $2(3.4)$ & \\
\hline Enzalutamide only & $7(9.9)$ & $1(7.7)$ & $6(10.4)$ & \\
\hline Abiraterone/Cabazitaxel & $1(1.4)$ & 0 & $1(1.7)$ & \\
\hline Abiraterone/Enzalutamide & $4(5.6)$ & 0 & $4(6.9)$ & \\
\hline Abiraterone/Cabazitaxel/Enzalutamide & $1(1.4)$ & 0 & $1(1.7)$ & \\
\hline
\end{tabular}

Data are presented as means $\pm \mathrm{SD}$ [median, range] or number $(\%)$.

$\mathrm{SD}$, standard deviation; CRPC, castration-resistant prostate cancer; PSA, prostate specific antigen; ADT, androgen deprivation therapy; HIFU, high-intensity focused ultrasound. 


\begin{tabular}{|c|c|c|c|c|c|c|c|c|c|c|c|c|c|c|c|c|c|c|c|}
\hline \multicolumn{2}{|c|}{ Case } & 1 & 2 & 3 & 4 & 5 & 6 & 7 & 8 & 9 & 10 & 11 & 12 & 13 & 14 & 15 & 16 & 17 & 18 \\
\hline \multirow{2}{*}{ IHC } & ERG & $\mathbf{N}$ & $\mathbf{N}$ & $\mathbf{N}$ & $\mathbf{N}$ & $\mathbf{N}$ & $\mathbf{N}$ & $\mathbf{N}$ & $\mathbf{N}$ & $\mathbf{P}$ & $\mathbf{N}$ & $\mathbf{N}$ & $\mathbf{N}$ & $\mathbf{N}$ & $\mathbf{N}$ & $\mathbf{N}$ & $\mathbf{N}$ & $\mathbf{N}$ & $\mathbf{N}$ \\
\hline & SOX9 & $\mathbf{P}$ & $\mathbf{P}$ & $\mathbf{P}$ & $\mathbf{P}$ & $\mathbf{P}$ & $\mathbf{P}$ & $\mathbf{P}$ & $\mathbf{P}$ & $\mathbf{P}$ & $\mathbf{P}$ & $\mathbf{P}$ & $\mathbf{N}$ & $\mathbf{N}$ & $\mathbf{P}$ & $\mathbf{N}$ & $\mathbf{P}$ & $\mathbf{P}$ & $\mathbf{P}$ \\
\hline \multicolumn{2}{|c|}{ Case } & 19 & 20 & 21 & 22 & 23 & 24 & 25 & 26 & 27 & 28 & 29 & 30 & 31 & 32 & 33 & 34 & 35 & 36 \\
\hline \multirow{2}{*}{ IHC } & ERG & $\mathbf{P}$ & $\mathbf{P}$ & $\mathbf{P}$ & $\mathbf{P}$ & $\mathbf{P}$ & $\mathbf{N}$ & $\mathbf{N}$ & $\mathbf{N}$ & $\mathbf{N}$ & $\mathbf{N}$ & $\mathbf{P}$ & $\mathbf{N}$ & $\mathbf{N}$ & $\mathbf{P}$ & $\mathbf{N}$ & $\mathbf{N}$ & $\mathbf{N}$ & $\mathbf{N}$ \\
\hline & SOX9 & $\mathbf{P}$ & $\mathbf{P}$ & $\mathbf{P}$ & $\mathbf{P}$ & $\mathbf{P}$ & $\mathbf{P}$ & $\mathbf{P}$ & $\mathbf{P}$ & $\mathbf{P}$ & $\mathbf{P}$ & $\mathbf{P}$ & $\mathbf{P}$ & $\mathbf{P}$ & $\mathbf{P}$ & $\mathbf{P}$ & $\mathbf{P}$ & $\mathbf{P}$ & $\mathbf{P}$ \\
\hline \multicolumn{2}{|c|}{ Case } & 37 & 38 & 39 & 40 & 41 & 42 & 43 & 44 & 45 & 46 & 47 & 48 & 49 & 50 & 51 & 52 & 53 & 54 \\
\hline \multirow{2}{*}{ IHC } & ERG & $\mathbf{N}$ & $\mathbf{N}$ & $\mathbf{N}$ & $\mathbf{N}$ & $\mathbf{N}$ & $\mathbf{N}$ & $\mathbf{N}$ & $\mathbf{P}$ & $\mathbf{P}$ & $\mathbf{N}$ & $\mathbf{N}$ & $\mathbf{N}$ & $\mathbf{N}$ & $\mathbf{N}$ & $\mathbf{N}$ & $\mathbf{N}$ & $\mathbf{N}$ & $\mathbf{N}$ \\
\hline & SOX9 & $\mathbf{P}$ & $\mathbf{P}$ & $\mathbf{N}$ & $\mathbf{N}$ & $\mathbf{P}$ & $\mathbf{P}$ & $\mathbf{P}$ & $\mathbf{P}$ & $\mathbf{P}$ & $\mathbf{P}$ & $\mathbf{P}$ & $\mathbf{P}$ & $\mathbf{N}$ & $\mathbf{P}$ & $\mathbf{N}$ & $P$ & $\mathbf{P}$ & $\mathbf{P}$ \\
\hline \multicolumn{2}{|c|}{ Case } & 55 & 56 & 57 & 58 & 59 & 60 & 61 & 62 & 63 & 64 & 65 & 66 & 67 & 68 & 69 & 70 & 71 & \\
\hline \multirow{2}{*}{ IHC } & ERG & $\mathbf{N}$ & $\mathbf{N}$ & $\mathbf{N}$ & $\mathbf{P}$ & $\mathbf{N}$ & $\mathbf{N}$ & $\mathbf{N}$ & $\mathbf{N}$ & $\mathbf{N}$ & $\mathbf{N}$ & $\mathbf{N}$ & $\mathbf{N}$ & $\mathbf{N}$ & $\mathbf{N}$ & $\mathbf{P}$ & $\mathbf{P}$ & $\mathbf{N}$ & \\
\hline & SOX9 & $\mathbf{P}$ & $\mathbf{P}$ & $\mathbf{N}$ & $\mathbf{P}$ & $\mathbf{P}$ & $\mathbf{P}$ & $\mathbf{P}$ & $\mathbf{P}$ & $\mathbf{P}$ & $\mathbf{P}$ & $\mathbf{P}$ & $\mathbf{P}$ & $\mathbf{P}$ & $\mathbf{P}$ & $\mathbf{P}$ & $\mathbf{P}$ & $\mathbf{N}$ & \\
\hline
\end{tabular}

Figure 1: Correlation between IHC-measured ERG and SOX9 expression in 71 mCRPC patients (P, positive; N, negative).

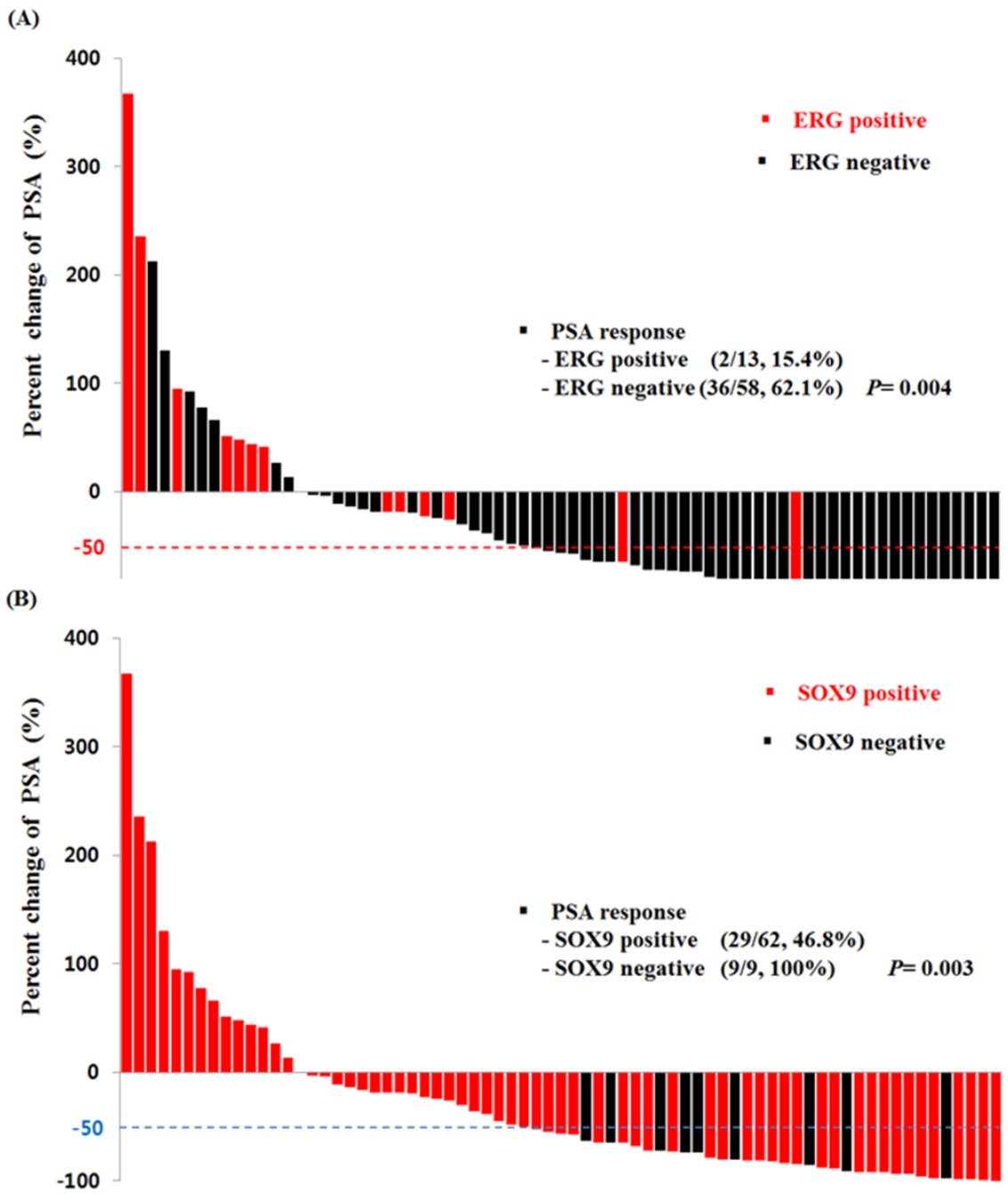

Figure 2: Waterfall plot of PSA levels in response to docetaxel treatment according to A. ERG and B. SOX9 expression. 
In comparison to previous studies [10, 15], our study focused on detecting ERG expression rather than the TMPRESS2-ERG rearrangement. Park et al [17] found IHC detection of ERG to have high sensitivity, specificity and accuracy in the assessment of TMPRESS2-ERG fusion status, and another study found that ERG IHC expression was congruent with the TMPRESS2-ERG fusion [18]. These results were further validated by a larger cohort study that the ERG IHC results could be used as a simple and accurate surrogate for TMPRESS2-ERG fusion status detection [19]. Previous studies, however, have highlighted the different clinical implications that exist for genetic alterations in CTCs vs. tissue [15], thus it has not yet been clearly determined whether tissue or blood would provide the most clinically useful ERG IHC results.

In addition, our study showed that expression of SOX9, a known downstream effector of ERG, was also correlated with a lower PSA-PFS, C/R-PFA and OS after docetaxel treatment in $\mathrm{MCRPC}$ patients. During fetal growth, it is well known that SOX9 is essential for prostate development, therefore a prostate-specific SOX9 knockout model results in profoundly defective prostate morphogenesis [20]. In the adult prostate, SOX9 plays an essential role in preserving the luminal epithelium [21]. However, recent studies examining the role of SOX9 in prostate cancer showed that overexpression was associated with a higher Gleason score [22], cancer progression and invasion [23]. In xenograft models of prostate cancer, increased expression of SOX9 causes cancer growth, invasion and angiogenesis, while silencing of SOX9 dramatically decreases tumor growth [14, 23].

Wang H. et al [21] reported that SOX9 expression was further increased in patients with mCRPC. In our study, all ERG-positive patients were also SOX9 positive and, interestingly, 49 of $58(84.5 \%)$ patients were negative for ERG but positive for SOX9. A possible explanation for this is the regulation of SOX9 by other pathways, including the Wnt/beta-catenin or MAP kinase pathways $[13,21]$. In addition, in vitro studies, SOX9 expression is suppressed by androgens in ERG-negative prostate cancer cells, therefore ADT may actually induce SOX9 expression in ERG-negative patients [14, 22]. Remarkably, 9 patients with both ERG and SOX9 negativity showed more favorable clinical outcomes after docetaxel treatment when compared to the other subgroups. Therefore, SOX9 expression could be used as biomarkers of the activation of other pathways known to provoke prostate cancer progression and of docetaxel response together with the results of ERG IHC [14].

Despite the clinical implications, our study had several limitations that need to be considered for interpretation. Foremost, this study was retrospective in design, conducted at a single institution and included a relatively small patient population, thus raising concern
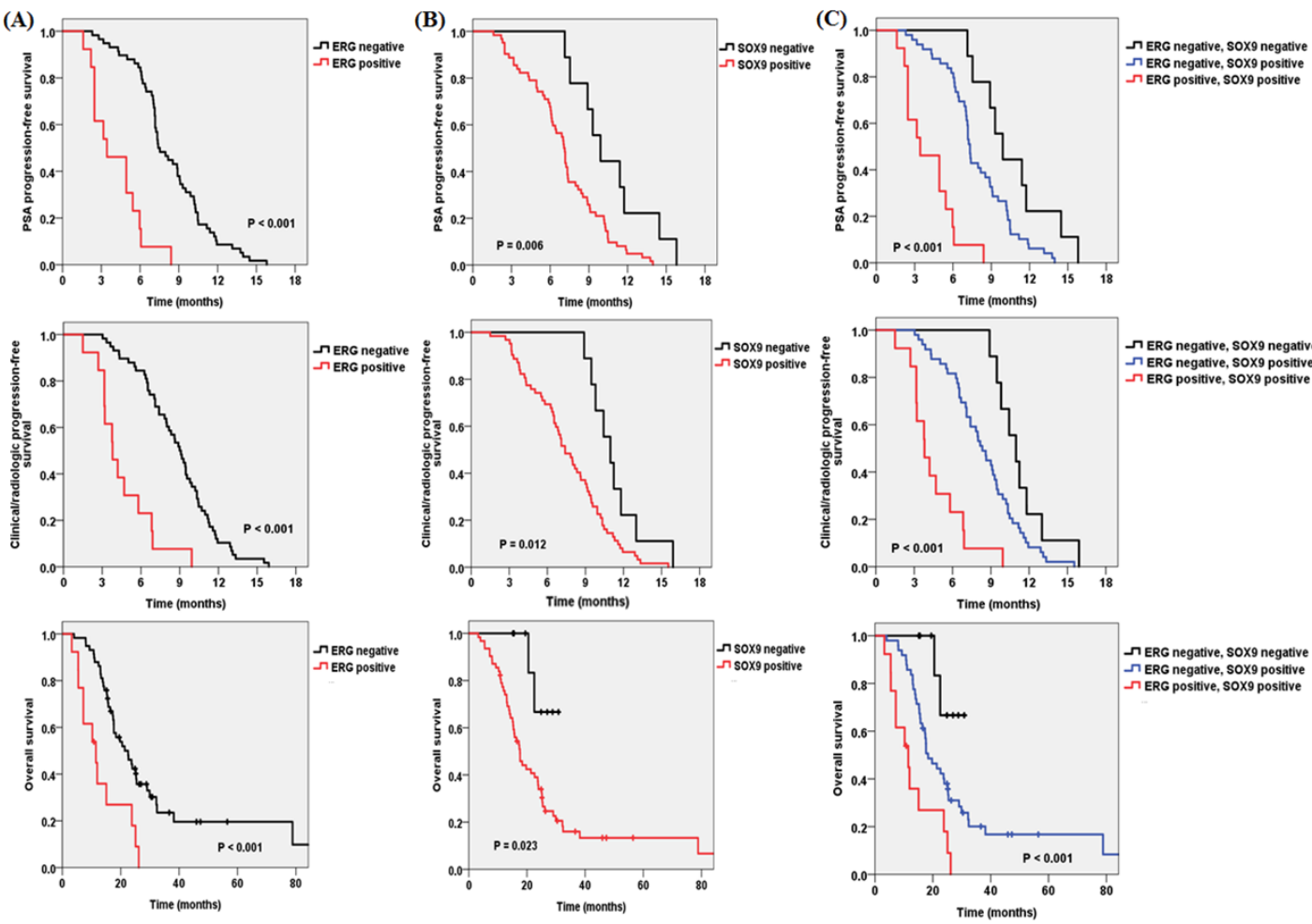

Figure 3: Kaplan-Meier analysis depicting PSA progression-free survival, clinical/radiologic progression-free survival and overall survival according to A. ERG, B. SOX9 and $\mathbf{C}$. ERG and SOX9 expression. 
Table 2: Multivariate Cox proportional hazard regression analyses in the prediction of PSA-progression free survival (PFS), C/R-PFS and overall survival (OS) in mCRPC patients

\begin{tabular}{|c|c|c|c|c|c|c|c|c|c|}
\hline \multirow{2}{*}{ Variables } & \multicolumn{3}{|c|}{ PSA-PFS } & \multicolumn{3}{|c|}{ C/R-PFS } & \multicolumn{3}{|c|}{ OS } \\
\hline & HR & $95 \%$ CI & $p$ & HR & $95 \% \mathrm{CI}$ & $p$ & HR & $95 \%$ CI & $p$ \\
\hline \multicolumn{10}{|l|}{ Age } \\
\hline$\leq 65.0$ & Ref & & & Ref & & & Ref & & \\
\hline$>65.0$ & 0.76 & $0.46-1.28$ & 0.306 & 0.91 & $0.54-1.53$ & 0.715 & 0.79 & $0.45-1.39$ & 0.420 \\
\hline \multicolumn{10}{|c|}{ PSA nadir after ADT } \\
\hline$\leq 1.0$ & Ref & & & Ref & & & Ref & & \\
\hline$>1.0$ & 1.13 & $0.68-1.88$ & 0.635 & 0.97 & $0.59-1.61$ & 0.920 & 0.98 & $0.55-1.72$ & 0.934 \\
\hline \multicolumn{10}{|c|}{ Gleason score } \\
\hline 7 & Ref & & & Ref & & & Ref & & \\
\hline $8-10$ & 0.56 & $0.27-1.17$ & 0.124 & 0.63 & $0.30-1.32$ & 0.221 & 1.08 & $0.46-2.53$ & 0.863 \\
\hline \multicolumn{10}{|c|}{ Metastatic volume } \\
\hline Low & Ref & & & Ref & & & Ref & & \\
\hline High & 1.57 & $0.89-2.78$ & 0.122 & 2.31 & $1.32-4.04$ & 0.003 & 2.88 & $1.44-5.76$ & 0.003 \\
\hline \multicolumn{10}{|l|}{ ERG } \\
\hline Negative & Ref & & & Ref & & & Ref & & \\
\hline Positive & 6.00 & $\begin{array}{l}2.96- \\
12.16\end{array}$ & $<0.001$ & 5.50 & $\begin{array}{l}2.68- \\
11.29\end{array}$ & $<0.001$ & 3.31 & $1.66-6.64$ & 0.001 \\
\hline \multicolumn{10}{|l|}{ SOX9 } \\
\hline Negative & Ref & & & Ref & & & Ref & & \\
\hline Positive & 2.75 & $1.19-6.32$ & 0.018 & 2.44 & $1.12-5.30$ & 0.025 & 4.30 & $\begin{array}{l}1.02- \\
18.16\end{array}$ & 0.047 \\
\hline
\end{tabular}

PSA-PFS, prostate specific antigen progression-free survival; C/R-PFS, clinical/radiologic progression-free survival; OS, overall survival; HR, hazard ratio; CI, confidence interval; ADT, androgen deprivation therapy.

for selection bias. However, this is the largest study to analyze the correlation between IHC-measured ERG and SOX9 and docetaxel response, as evaluated by the PSA response, PSA-PFS, C/R-PFA and OS. Second, when interpreting IHC results, we only considered intensity and did not apply other interpretation methods. Since there are no objective guidelines for interpretation, there may be a discrepancy in the results. Finally, other important prognostic factors, such as alkaline phosphatase and lactic dehydrogenase, were not considered due to lack of available information.

In conclusion, our result indicated that IHC-detected ERG and SOX9 expression is significantly associated with lower PSA-PFS, C/R-PFS and OS in patients with mCRPC treated with docetaxel. Therefore, they could be used as potential biomarkers for prediction to docetaxel treatment in mCRPC patients. Further large, prospective clinical trials are necessary to confirm our results.

\section{MATERIALS AND METHODS}

\section{Study population and data collection}

A retrospective study was conducted on 78 patients who were diagnosed with $\mathrm{mCRPC}$ and treated with docetaxel $\left(75 \mathrm{mg} / \mathrm{m}^{2}\right.$ intravenously, every 3 weeks $)$ between 2001 and 2013. We excluded 7 patients who had tissue samples collected after docetaxel treatment. The tissues of the remaining 71 patients were obtained from prostate biopsy specimens at the time of diagnosis. Clinical information including follow-up data after docetaxel treatment was retrieved from the patients' medical charts. In regards to metastatic status, high volume disease was defined as the existence of visceral metastases or having more than 4 bone lesions, one of which was present beyond the vertebral bodies or pelvis [24]. To assess the therapeutic response, the serum PSA was measured after each treatment and a computed tomography (CT) and/or 
bone scan was performed after every third treatment. This study was carried out with the approval of the institutional review board committee at our institution.

\section{Response evaluation}

After docetaxel treatment, we evaluated the PSA response rate, PSA progression-free survival (PSA-PFS), clinical/radiologic PFS (C/R-PFS) and the overall survival (OS). Treatment response to docetaxel was assessed based on the recommendations of the Prostate Cancer Working Group 2 (PCWG2) [25]. The PSA response was measured using the percent change of the PSA level from baseline to 12 weeks post-treatment, and a therapeutic response was defined as more than a $50 \%$ decline from baseline maintained over at least 4 weeks. The PSA-PFS, C/R-PFS and OS were estimated based on the time between the initial treatment and PSA progression, $\mathrm{C} / \mathrm{R}$ progression and death or last visit. PSA progression was defined as an increase in the PSA more than $25 \%$ (at least $2 \mathrm{ng} / \mathrm{ml}$ ) from the nadir that persisted for at least 3 weeks. Clinical progression was defined as a new onset or aggravation of cancer-related symptoms or a higher analgesic requirement [15]. Radiological progression was defined as two or more new lesions confirmed on a bone scan or evidence of progression on a CT scan, as per the recommendations of the Response Evaluation Criteria in Solid Tumor version 1.1 (RECIST v1.1) $[15,26]$.

\section{TMA construction}

Typical formalin-fixed paraffin-embedded (FFPE) tissue blocks were obtained from hematoxylin-eosin stained slides for TMA preparation. Two, 2-mm tissue cores were taken from the donor blocks with the use of a manual tissue microarrayer (ISU ABXIS, Seoul, Republic of Korea) and placed into a recipient TMA block.

\section{IHC detection of ERG and SOX9 and interpretation}

Paraffin-embedded TMA sections $4 \mu \mathrm{m}$ thick were mounted on slides to determine ERG and SOX9 expression levels. The IHC for ERG was performed on a BenchMark XT automated stainer (Roche/Ventana Medical Systems, Tucson, Arizona, USA) using an ERG rabbit monoclonal antibody (EPR3864, Epitomics, Burlingame, California, USA; dilution 1:100) according to the manufacturer's instructions. Vascular endothelial cells and normal horse serum replaced by primary antibodies were used as positive and negative controls, respectively. IHC of SOX9 was performed as follows: First, the endogenous peroxidase activity was blocked by incubating each section in $0.3 \%$ hydrogen peroxide. Antigens were then retrieved by heating the sections in Target Retrieval Solution ( $\mathrm{pH}$ 9.0) (DAKO, Denmark) at $95^{\circ} \mathrm{C}$ for 20 minutes. The sections were incubated overnight at $4^{\circ} \mathrm{C}$ with primary mouse monoclonal antibodies against SOX9 (Abnova, Taipei, Taiwan), followed by a 1-hour incubation at room temperature (RT) with ChemMate ${ }^{\mathrm{TM}} \mathrm{DAKO}$ EnVision TM/HRP (DAKO, Denmark) and an additional hour at RT with DakoCytomation TechMate ${ }^{\mathrm{TM}}$ (DAKO, Denmark).

The IHC results were interpreted by an experienced genitourinary pathologist (G.Y. K). The intensities of ERG and SOX9 expression in tumor cells were scored using a four-tiered grading system, which was as follows: negative ( 0 ; no staining), weak (1+; visible only at high magnification), moderate $(2+$; visible at low magnification) or strong (3+; prominent at low magnification) (Figure 4). A sample with more than 5\% of total stained area scoring $2+$ or $3+$ in intensity was considered to be positive [27].

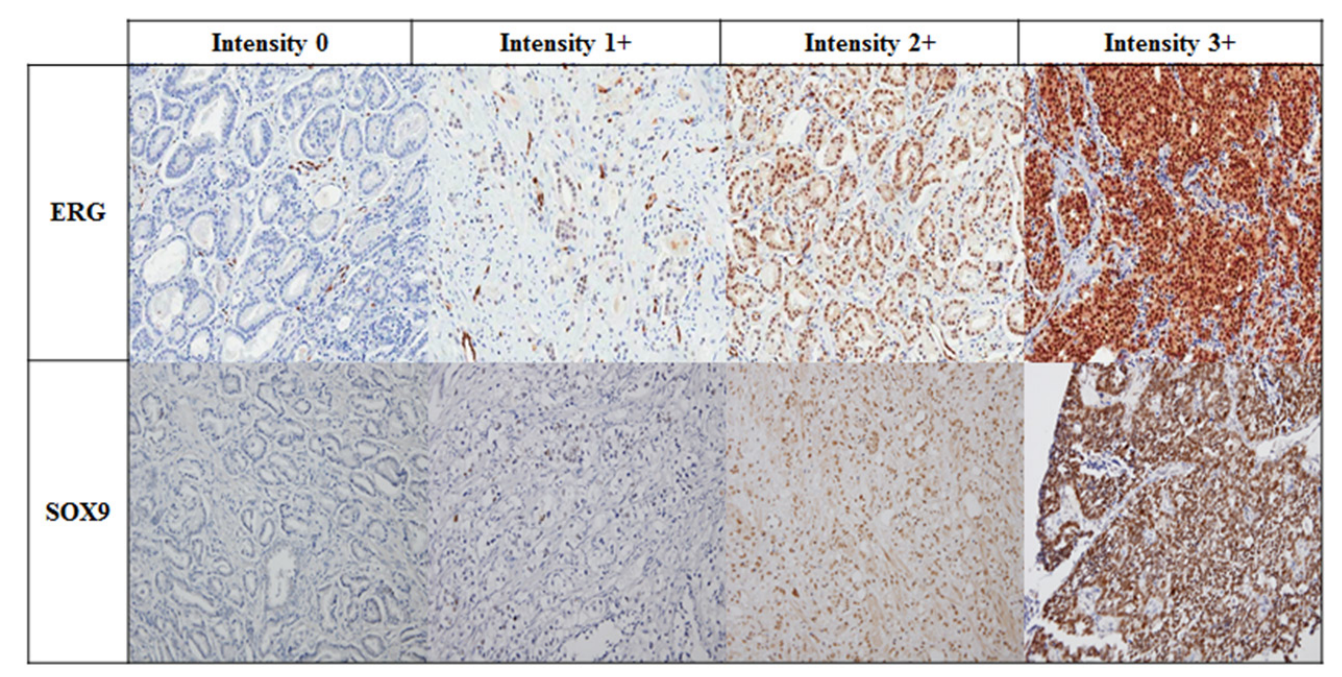

Figure 4: Representative images of ERG and SOX9 detection by IHC in mCRPC patients according to intensity (Magnification x 200). 


\section{Statistical analysis}

Continuous and categorical variables were described as means (standard deviation, range) and absolute values (percentage). An independent $t$-test and Fisher's exact test or linear by linear association were used to compare clinical characteristics. Individual PSA responses were depicted by a waterfall plot and compared using the Fisher's exact test. Kaplan-Meier survival curves were constructed to illustrate the PSA-PFS, C/R-PFS and OS according to the expression patterns of ERG and SOX9, and a log rank test was employed to compare patient subgroups. Univariate and multivariate Cox proportional hazard models were utilized to estimate associations between the PSA-PFS, C/R-PFS, OS and risk factors of interest. All statistical analyses were performed with IBM SPSS version 20.0 (IBM Corp. Armonk, NY, USA). Two-tailed $p$ values $<0.05$ were considered statistically significant.

\section{ACKNOWLEDGMENTS}

Author's contribution: WS, BCJ and HML designed the study. WS and JEL collected data. WS, GYK, JHK and BCJ did statistical analysis and interpreted data. WS drafted the manuscript. GYK, JHK, HGJ, SIS, SSJ, HYC, BCJ and HML did the critical revision of manuscript. BCJ and HML supervised this study. All authors have made substantial contributions to the intellectual contents of this manuscript and approved the submission.

\section{CONFLICTS OF INTEREST}

The authors declare no conflicts of interest.

\section{FUNDING}

This study was supported by grants from Korean Foundation for Cancer Research (CB-2011-04-01).

\section{REFERENCES}

1. Heidenreich A, Pfister D, Merseburger A, Bartsch G and German Working Group on Castration-Resistant Prostate C. Castration-resistant prostate cancer: where we stand in 2013 and what urologists should know. Eur Urol. 2013; 64:260-265.

2. Heidenreich A, Bastian PJ, Bellmunt J, Bolla M, Joniau S, van der Kwast T, Mason M, Matveev V, Wiegel T, Zattoni F, Mottet $\mathrm{N}$ and European Association of U. EAU guidelines on prostate cancer. Part II: Treatment of advanced, relapsing, and castration-resistant prostate cancer. Eur Urol. 2014; 65:467-479.

3. Petrylak DP, Tangen CM, Hussain MH, Lara PN, Jr., Jones JA, Taplin ME, Burch PA, Berry D, Moinpour C, Kohli
M, Benson MC, Small EJ, Raghavan D, Crawford ED. Docetaxel and estramustine compared with mitoxantrone and prednisone for advanced refractory prostate cancer. $\mathrm{N}$ Engl J Med. 2004; 351:1513-1520.

4. Tannock IF, de Wit R, Berry WR, Horti J, Pluzanska A, Chi KN, Oudard S, Theodore C, James ND, Turesson I, Rosenthal MA, Eisenberger MA and Investigators TAX. Docetaxel plus prednisone or mitoxantrone plus prednisone for advanced prostate cancer. N Engl J Med. 2004; 351:1502-1512.

5. Saad F, Miller K. Treatment options in castration-resistant prostate cancer: current therapies and emerging docetaxelbased regimens. Urol Oncol. 2014; 32:70-79.

6. Marin-Aguilera M, Codony-Servat J, Kalko SG, Fernandez PL, Bermudo R, Buxo E, Ribal MJ, Gascon P, Mellado B. Identification of docetaxel resistance genes in castrationresistant prostate cancer. Mol Cancer Ther. 2012; 11:329-339.

7. Khemlina G, Ikeda S, Kurzrock R. Molecular landscape of prostate cancer: implications for current clinical trials. Cancer Treat Rev. 2015; 41:761-766.

8. Lorente D, De Bono JS. Molecular alterations and emerging targets in castration resistant prostate cancer. Eur J Cancer. 2014; 50:753-764.

9. Semenas J, Allegrucci C, Boorjian SA, Mongan NP, Persson JL. Overcoming drug resistance and treating advanced prostate cancer. Curr Drug Targets. 2012; 13:1308-1323.

10. Galletti G, Matov A, Beltran H, Fontugne J, Miguel Mosquera J, Cheung C, MacDonald TY, Sung M, O'Toole S, Kench JG, Suk Chae S, Kimovski D, Tagawa ST, Nanus DM, Rubin MA, Horvath LG, Giannakakou P, Rickman DS. ERG induces taxane resistance in castration-resistant prostate cancer. Nat Commun. 2014; 5:5548.

11. Roudier MP, Winters BR, Coleman I, Lam HM, Zhang X, Coleman R, Chery L, True LD, Higano CS, Montgomery B, Lange PH, Snyder LA, Srivastava S, Corey E, Vessella RL, Nelson PS, Uren A, Morrissey C. Characterizing the molecular features of ERG-positive tumors in primary and castration resistant prostate cancer. Prostate. 2016; 76:810-822.

12. Kavallaris M. Microtubules and resistance to tubulinbinding agents. Nat Rev Cancer. 2010; 10:194-204.

13. Yuan X, Cai C, Chen S, Chen S, Yu Z, Balk SP. Androgen receptor functions in castration-resistant prostate cancer and mechanisms of resistance to new agents targeting the androgen axis. Oncogene. 2014; 33:2815-2825.

14. Cai C, Wang H, He HH, Chen S, He L, Ma F, Mucci L, Wang Q, Fiore C, Sowalsky AG, Loda M, Liu XS, Brown $\mathrm{M}$, Balk SP, Yuan X. ERG induces androgen receptormediated regulation of SOX9 in prostate cancer. J Clin Invest. 2013; 123:1109-1122.

15. Reig O, Marin-Aguilera M, Carrera G, Jimenez N, Pare L, Garcia-Recio S, Gaba L, Pereira MV, Fernandez P, Prat A, Mellado B. TMPRSS2-ERG in Blood and Docetaxel 
Resistance in Metastatic Castration-resistant Prostate Cancer. Eur Urol. 2016.

16. Tomlins SA, Laxman B, Varambally S, Cao X, Yu J, Helgeson BE, Cao Q, Prensner JR, Rubin MA, Shah RB, Mehra R, Chinnaiyan AM. Role of the TMPRSS2ERG gene fusion in prostate cancer. Neoplasia. 2008; 10:177-188.

17. Park K, Tomlins SA, Mudaliar KM, Chiu YL, Esgueva R, Mehra R, Suleman K, Varambally S, Brenner JC, MacDonald T, Srivastava A, Tewari AK, Sathyanarayana U, Nagy D, Pestano G, Kunju LP, Demichelis F, Chinnaiyan AM, Rubin MA. Antibody-based detection of ERG rearrangement-positive prostate cancer. Neoplasia. 2010; 12:590-598.

18. Furusato B, Tan SH, Young D, Dobi A, Sun C, Mohamed AA, Thangapazham R, Chen Y, McMaster G, Sreenath T, Petrovics G, McLeod DG, Srivastava S, Sesterhenn IA. ERG oncoprotein expression in prostate cancer: clonal progression of ERG-positive tumor cells and potential for ERG-based stratification. Prostate Cancer Prostatic Dis. 2010; 13:228-237.

19. Chaux A, Albadine R, Toubaji A, Hicks J, Meeker A, Platz EA, De Marzo AM, Netto GJ. Immunohistochemistry for ERG expression as a surrogate for TMPRSS2-ERG fusion detection in prostatic adenocarcinomas. Am J Surg Pathol. 2011; 35:1014-1020.

20. Huang Z, Hurley PJ, Simons BW, Marchionni L, Berman DM, Ross AE, Schaeffer EM. Sox9 is required for prostate development and prostate cancer initiation. Oncotarget. 2012; 3:651-663. doi: 10.18632/oncotarget.531.

21. Wang H, McKnight NC, Zhang T, Lu ML, Balk SP, Yuan $\mathrm{X}$. SOX9 is expressed in normal prostate basal cells and regulates androgen receptor expression in prostate cancer cells. Cancer Res. 2007; 67:528-536.

22. Thomsen MK, Ambroisine L, Wynn S, Cheah KS, Foster CS, Fisher G, Berney DM, Moller H, Reuter VE,
Scardino P, Cuzick J, Ragavan N, Singh PB, Martin FL, Butler CM, Cooper CS, Swain A, Transatlantic Prostate G. SOX9 elevation in the prostate promotes proliferation and cooperates with PTEN loss to drive tumor formation. Cancer Res. 2010; 70:979-987.

23. Wang H, Leav I, Ibaragi S, Wegner M, Hu GF, Lu ML, Balk SP, Yuan X. SOX9 is expressed in human fetal prostate epithelium and enhances prostate cancer invasion. Cancer Res. 2008; 68:1625-1630.

24. Sweeney CJ, Chen YH, Carducci M, Liu G, Jarrard DF, Eisenberger M, Wong YN, Hahn N, Kohli M, Cooney MM, Dreicer R, Vogelzang NJ, Picus J, Shevrin D, Hussain M, Garcia JA, DiPaola RS. Chemohormonal Therapy in Metastatic Hormone-Sensitive Prostate Cancer. N Engl J Med. 2015; 373:737-746.

25. Scher HI, Halabi S, Tannock I, Morris M, Sternberg CN, Carducci MA, Eisenberger MA, Higano C, Bubley GJ, Dreicer R, Petrylak D, Kantoff P, Basch E, Kelly WK, Figg WD, Small EJ, Beer TM, Wilding G, Martin A, Hussain M, Prostate Cancer Clinical Trials Working G. Design and end points of clinical trials for patients with progressive prostate cancer and castrate levels of testosterone: recommendations of the Prostate Cancer Clinical Trials Working Group. J Clin Oncol. 2008; 26:1148-1159.

26. Eisenhauer EA, Therasse P, Bogaerts J, Schwartz LH, Sargent D, Ford R, Dancey J, Arbuck S, Gwyther S, Mooney M, Rubinstein L, Shankar L, Dodd L, Kaplan R, Lacombe D, Verweij J. New response evaluation criteria in solid tumours: revised RECIST guideline (version 1.1). Eur J Cancer. 2009; 45:228-247.

27. Schaefer G, Mosquera JM, Ramoner R, Park K, Romanel A, Steiner E, Horninger W, Bektic J, Ladurner-Rennau M, Rubin MA, Demichelis F, Klocker H. Distinct ERG rearrangement prevalence in prostate cancer: higher frequency in young age and in low PSA prostate cancer. Prostate Cancer Prostatic Dis. 2013; 16:132-138. 